\title{
Custo do Estoque para Atendimento da Demanda a partir da Distribuição Normal
}

\author{
Jessica Syrio Callefi \\ Doutorado em andamento em Engenharia de Produção pela Escola de Engenharia de \\ São Carlos/ \\ Universidade de São Paulo - EESC/USP \\ Avenida Guaiapó, 3144. Maringá/PR. CEP: 87047-402 \\ E-mail: jessica.callefi@gmail.com
}

\section{RESUMO}

As discussões a respeito do trade-off estoque versus atendimento da demanda não são recentes. Diversos estudos apontam a importância do dimensionamento correto do estoque para que não se perca clientes por falta de itens em estoque e, ainda, que este não tenha um custo muito elevado para a empresa. O presente artigo tem como objetivo definir qual a variação em relação à média mais vantajosa em termos de estoque e de atendimento ao cliente para a empresa ABC. Para isso, utilizou-se o Gráfico de Pareto para eleger os produtos mais importantes para a empresa e, posteriormente, utilizou-se a distribuição normal para analisar os valores da demanda em relação aos seus custos. Para este estudo de caso, observa-se que a variação de 2 desvios em relação à média foi considerada a mais satisfatória para a empresa, visto que o aumento de custos de estoque é suportado pela fidelização dos clientes. Como principais resultados obtidos para a empresa, citam-se: maior clareza sobre a demanda dos seus principais produtos e seus custos, possibilidade de redução de estoques segura e a melhora na organização do trabalho dos setores de programação da produção, compras de matéria-prima e vendas. Conclui-se que é possível utilizar a distribuição normal estatística para definir lotes de estoque como um meio de fácil usabilidade para os gestores analisarem seus dados, compararem com o aumento de custos relativos ao atendimento de $68,3 \%, 95,4 \%$ ou $99,7 \%$ de sua demanda e tomarem suas decisões.

Palavras-chave: Estoque. Demanda. Distribuição normal.

\section{Cost of the Stock for Customer Service from Normal Distribution}

\begin{abstract}
Discussions regarding the stock-versus-demand trade-off are not recent. Several studies point out the importance of the correct dimensioning of the inventory so that clients are not lost due to the lack of items in stock and that this inventory does not have a very high cost for the company. The purpose of this article is to define the variation from the most advantageous average in terms of inventory and customer service for $A B C$
\end{abstract}


Company. For this, the Pareto Chart was used to choose the most important products for the company and, later, the normal distribution was used to analyze the values of the demand in relation to its costs. For this case study, it is observed that the variation of 2 deviations from the average was considered the most satisfactory for the company, since the increase of inventory costs is supported by customer loyalty. The main results obtained for the company are: greater clarity on the demand for its main products and its costs, the possibility of safe inventory reduction, and the improvement in the work organization of the sectors of production scheduling, premium and sales. It is concluded that it is possible to use the normal statistical distribution to define stock lots as a means of easy usability for managers to analyze their data, compare with the increase of costs related to the attendance of $68.3 \%, 95.4 \%$ or $99.7 \%$ of their demand and make their decisions.

Key words: Stock. Demand. Normal curve.

\section{INTRODUÇÃO}

Com o passar dos anos, o domínio de mercados se tornou mais acirrado e os clientes mais exigentes. Esse panorama exige que as empresas trabalhem com o menor custo possível para que possam atender as exigências dos compradores, obter mais lucro, ou ainda, conseguirem sobreviver no mercado.

As necessidades dos clientes determinam a escolha da empresa fornecedora de determinados produtos e componentes. Os requisitos dos clientes podem ser: desempenho dos produtos, estética, marca, preço de mercado e, até mesmo, a disponibilidade do item a qualquer momento.

Focando-se na disponibilidade de produtos em estoque, tem-se que as empresas fornecedoras de produtos e componentes trabalham com o dilema do trade-off custo de manter estoque versus o custo de perder clientes por falta de estoque.

Diversas ferramentas de análise de dados auxiliam na visualização e, consequentemente, no entendimento deles para uma melhor tomada de decisões. $O$ Gráfico de Pareto e a análise da distribuição normal são exemplos de ferramentas amplamente utilizadas dentro das indústrias de manufatura atualmente, pois o Gráfico de Pareto auxilia na escolha dos itens ou problemas de maior impacto, e a distribuição 
normal possibilita que se tenha certeza sobre os valores que estariam dentro de determinadas faixas de variações em relação à média.

Neste ínterim e dentro da problemática de trade-off de estoque, esta pesquisa buscou listar os produtos mais relevantes para a empresa utilizada neste estudo e, a partir desses itens, analisar os dados de suas demandas em relação à faixa de variação da curva normal, analisando os custos provenientes do estoque, considerando três cenários, sendo eles 1, 2 e 3 desvios em relação à média. Desta forma, esta pesquisa buscou responder: qual a variação em relação à média mais vantajosa em termos de estoque e de atendimento ao cliente para a empresa $A B C$ ?

Assim, este trabalho justifica-se por buscar um equilíbrio entre o menor custo para a empresa, prezando pelo bom atendimento ao cliente, utilizando duas ferramentas de fácil usabilidade, o que pode contribuir com o campo prático a partir de uma técnica que permite a análise rápida e simples para tomadas de decisões.

No campo teórico, observa-se que diversas teorias, como EOQ (Economic Order Quantity), ROP (Reorder Point), análise da curva ABC e sistemas de MRP (Material Resource Planning) vêm sendo amplamente utilizadas para gestão de estoques, bem como já são consolidadas na academia científica (Huiskonen, 2001). Entretanto, ainda é possível encontrar níveis de estoque mais altos ou mais baixos do que os necessários nas empresas (Christopher, 2009). Dessa forma, a análise de custo do estoque pela quantidade a partir da variação da curva normal pode trazer novos entendimentos acerca do estoque.

\section{REFERENCIAL TEÓRICO}

\subsection{Estoque}

Os objetivos operacionais do estoque são: cobrir as mudanças previstas no suprimento e na demanda, proteger contra as incertezas e permitir a compra ou a produção econômica (Moreira, 2004). Ou ainda, prover uma oportunidade de obtenção 
de ganhos por meio de especulações, com o uso do estoque (Moura, 2001). Dessa forma, é primordial saber o que se deve estocar.

Para Botter e Fortuin (2000), os itens a serem armazenados são determinados pela sua criticidade, ou seja, quais serão as consequências de não haver determinado item em estoque quando o cliente o necessita. Assim, é importante que os gestores analisem também os riscos que, comumente, não são levados em consideração na gestão de estoques tradicional (Rogers, Ribeiro \& Rogers, 2004).

Outro fator primordial dentro da gestão de estoques é o custo, pois o estoque tem diversos custos associados a ele, como: custo do item, custo do pedido, custo unitário de manutenção e o custo da falta de estoque (Moreira, 2004). Portanto, há que se avaliar a necessidade de manter estoque do ponto de vista de seu impacto nos custos totais e sobre a receita de vendas (Christopher, 2009). Em outras palavras: pode ocorrer um aumento na receita de vendas com um aumento no nível de estoques; pode-se perder clientes pela falta de itens em estoque; ou ainda, os custos de manutenção de estoque podem se tornar tão elevados que acabam se tornando inviáveis de praticar. Assim, é bastante complexo avaliar até que ponto a prática do estoque é favorável (Christopher, 2009). Dessa forma, é necessário avaliar cada caso para decidir qual a melhor solução em estoque para a empresa fornecedora e seus clientes.

Nas últimas décadas, com o advento do Sistema Toyota de Produção, manter estoques além dos limites é considerado um desperdício que deve ser eliminado (Womack, Jones \& Roos, 2004). Entretanto, para implantar uma nova prática de gestão de estoques, é necessário olhar para toda a cadeia de fornecedores, de forma que as novas práticas auxiliem em uma melhora da entrega dos produtos e redução dos custos (Frazzon, Tortorella, Dávalos, Holtz, \& Coelho, 2017).

Portanto, novas formas de repensar o estoque estão surgindo, o que torna a discussão deste tema ainda mais relevante e demanda a utilização de novas ferramentas para a análise das quantidades em estoques. 


\subsection{Ferramentas de análise}

Segundo Martins e Domingues (2011), necessitamos de conhecimentos para compreender as informações numéricas produzidas; e os procedimentos, técnicas e métodos estatísticos são fundamentais para o auxílio à execução dessas tarefas, tanto para analisar e compreender os dados, quanto para prever o comportamento destes ao longo do tempo.

Assim, diversas ferramentas foram desenvolvidas para auxiliarem os gestores no controle e tomada de decisões, como as sete ferramentas da qualidade, que são: Folha de Verificação, Diagrama de Ishikawa, Fluxograma, Histograma, Gráfico de Dispersão, Diagrama de Pareto e Cartas de Controle (Montgomery \& Runger, 2003).

O gráfico de Pareto ou a Curva 80-20 auxilia no entendimento e na análise dos dados. O conceito 80-20 surge das observações de padrões em diversas empresas onde se sabe que os itens que apresentam as maiores quantidades de vendas representam cerca de $20 \%$ do rol de produtos da empresa (Ballou, 2004). Esse gráfico auxilia na compreensão de quais são os itens mais representativos dentro de um universo extenso de dados, de modo que o enfoque possa ser dado nos itens de maior impacto para a empresa.

O Gráfico de Pareto é utilizado amplamente em análises de estoques, como nos estudos de Silva Júnior e Costa (2014) e Ferrari e Rodrigues (2014), que utilizaram somente estas ferramentas para tomarem suas decisões em relação à política de estoques que estudaram. Porém, outros estudos como o de Carvalho e Moura Oliveria (2018) utilizaram esta ferramenta em conjunto com outras, neste caso o kanban.

Além do gráfico de Pareto, a análise da distribuição normal é amplamente utilizada para o controle de processo, onde se utilizam as Cartas de Controle. Entretanto, neste estudo, apenas realizou-se a análise da curva normal para mostrar a variação dos dados de demanda em relação à média, o qual é representado pelo termo sigma $(\sigma)$. Esta técnica foi utilizada para garantir um mínimo de previsibilidade no atendimento da demanda. 
De maneira prática, para medir a variação, é necessário calcular a média aritmética de uma população de N observações, a qual Martins \& Domingues (2011) define segundo a Equação 1.

$$
\mu=\frac{\sum X_{i}}{N}
$$

\section{Equação 1. Média aritmética de uma população.}

Segundo Martins e Domingues (2011), é necessário analisar os desvios (di) para medir a dispersão entre os dados em relação à média. A variância $\left(\sigma^{2}\right)$ de uma população com $\mathrm{N}$ medidas será igual à soma dos quadrados desses desvios $\left(\sum d_{i}^{2}\right)$, dividida por N, como segue na Equação 2:

$$
\sigma^{2}=\frac{\sum d_{i}^{2}}{N}=\frac{\sum\left(X_{i}-\mu\right)^{2}}{N}
$$

\section{Equação 2. Cálculo da variância de uma população.}

A Figura 1 apresenta o gráfico de distribuição normal, salientando a localização dos dados de média e dos desvios padrão no gráfico.

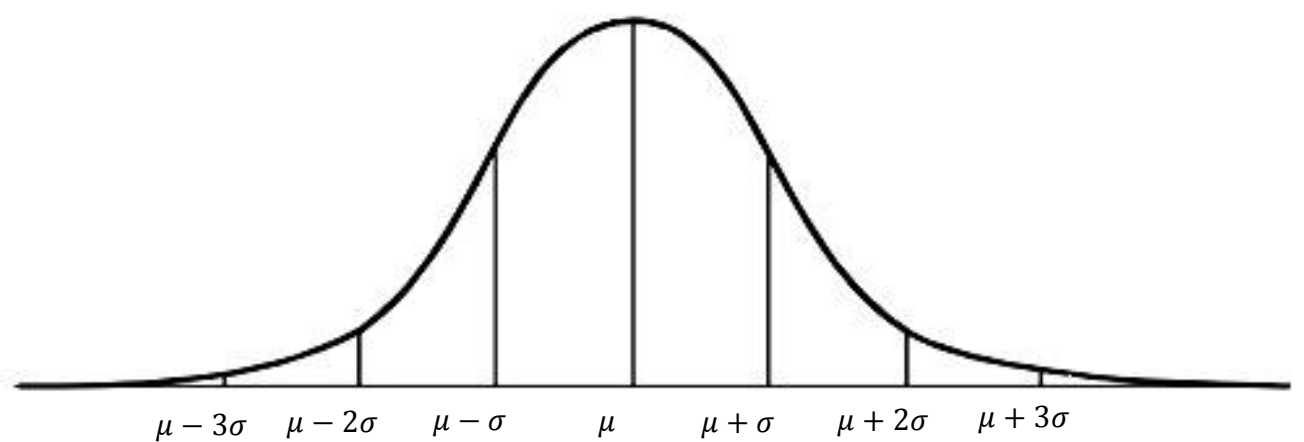

Figura 1. Gráfico da distribuição normal. 
Ainda segundo Christopher (2009), sabe-se que, em 68,3\% das vezes, a demanda estará na faixa de desvio padrão entre +1 e -1 em relação à média; em 95,4\% das vezes, a demanda estará na faixa de desvio padrão entre +2 e -2; e, em 99,7\%, estaria entre +3 e -3 em relação à média.

Para definir os limites dentro dos quais se considera que os dados estão sob controle e, portanto, seguem o comportamento normal estatístico, utilizou-se a equação 3 , que representa o limite superior de controle, e a equação 4 , que representa o limite inferior de controle (Montgomery \& Runger, 2003), onde $\mu$ é a média, $\sigma$ é o desvio padrão e $d$ representa a distância dos valores em relação à média. No caso desta pesquisa, os valores $-1,+1,-2,+2,-3$ e +3 foram utilizados como $d$.

$$
L S C=\mu+d \cdot \sigma
$$

Equação 3. Limite superior de Controle

$$
L I C=\mu-d . \sigma
$$

\section{Equação 4. Limite inferior de Controle}

O uso da curva normal permite que se tenha a confiabilidade para determinar se os dados estão sob controle estatístico e, portanto, estariam dentro de uma determinada faixa de valores específica.

\section{PROCEDIMENTOS METODOLÓGICOS}

Segundo Kauark, Manhães e Medeiros (2010), a natureza desta pesquisa se caracteriza como pesquisa aplicada, visto que tem o intuito de gerar conhecimentos para aplicação prática. A abordagem do problema utiliza-se da pesquisa quantitativa, pois se trata de análise de dados numéricos, com técnicas estatísticas. Ainda, o objetivo se utiliza da pesquisa descritiva, pois envolve o uso de técnicas padronizadas 
de coletas de dados e pretende entender o comportamento desses dados. $O$ procedimento técnico adotado é o estudo de caso (Yin, 2007).

Os dados para a realização deste estudo foram obtidos em uma empresa do setor metal-mecânico de pequeno porte, a qual opera no segmento de componentes para compressores de ar. A família de produtos utilizada para a análise neste estudo representa a maior parcela de rendimento desta empresa. Cada um dos produtos analisados apresenta demanda, valores e tamanhos diferentes. Para compor o estudo, utilizou-se um rol de sete produtos finais, dos quais a empresa não conhecia suas demandas e seus relativos custos de estoque.

Cada um desses sete produtos é composto por cinco componentes cada, os quais são montados no processo final. Cada um dos componentes passa por processos de fabricação distintos, como: injeção de plástico, usinagem, pintura e estamparia, conforme apresentado na Figura 2. Portanto, estabelecer-se um número para a demanda semanal é crucial para um bom funcionamento do setor de programação de produção e do próprio setor de manufatura da empresa.

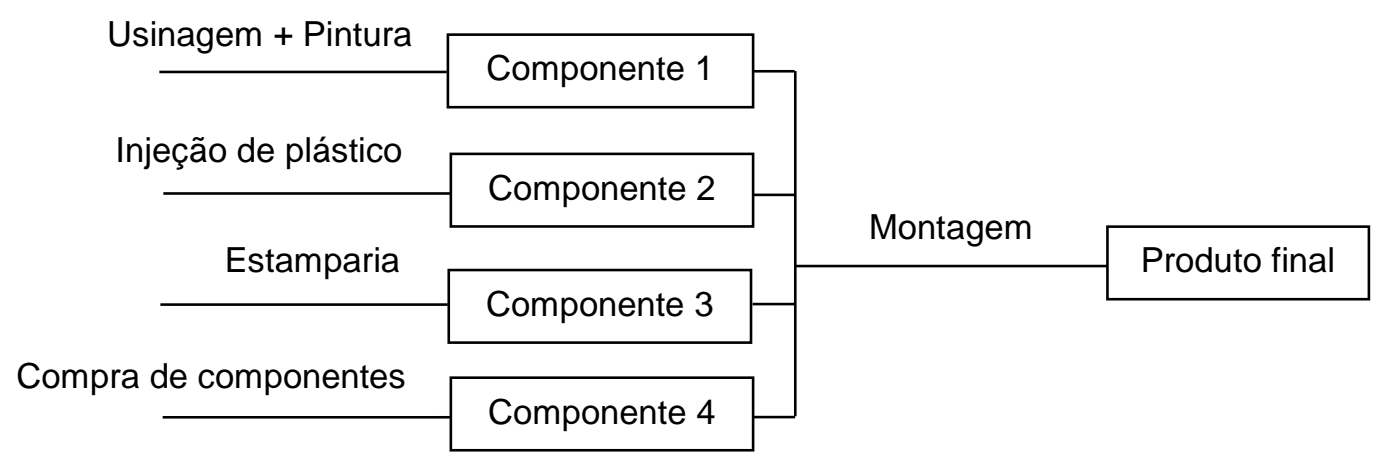

Figura 2. Componentes e processos padrão do produto analisado no estudo

Os dados do estudo abrangem os anos de 2011 a 2015 e foram obtidos a partir do banco de dados da própria empresa. Os dados coletados foram: a quantidade de itens vendidos, o valor unitário de cada item e a data de venda desses itens. Esses dados foram tabulados em planilhas eletrônicas de Excel, e as quantidades de itens 
vendidos foram agrupadas por semanas, pois o lote de produção é gerado para suprir a demanda semanal.

Por questão de sigilo concordado com a empresa, objeto de estudo, os dados de demanda, o tipo de produto, o nome e a localização da empresa não puderam ser divulgados, somente os resultados finais, conforme apresentado nas seções de resultado, discussões e conclusões.

Em posse dos dados coletados, estes foram organizados a partir do Gráfico de Pareto, onde ficou definido que, dentre os 7 produtos, somente 3 seriam analisados pela distribuição normal devido à sua representatividade para os rendimentos da empresa.

Depois disso, analisaram-se os dados desses 3 produtos, utilizando-se planilhas estatísticas, onde se excluíram os valores maiores ou menores que três desvios padrão em relação à média, considerando-se que esses valores são causas especiais e devem ter um tratamento diferente do tratamento padrão de estoque proposto neste estudo (Werkema, 1995). Um melhor detalhamento desses procedimentos é descrito na seção de resultados.

\section{RESULTADOS}

Primeiramente, plotou-se o Gráfico de Pareto em planilha do Excel e, posteriormente, realizou-se a análise do Gráfico de Pareto, no intuito de definir quais os produtos mais representativos para a empresa, tanto em relação à quantidade quanto em relação ao custo. Assim, multiplicaram-se as demandas ou itens vendidos pelos seus valores unitários e definiu-se a porcentagem correspondente de cada item. Os dados obtidos estão no Gráfico 1. 


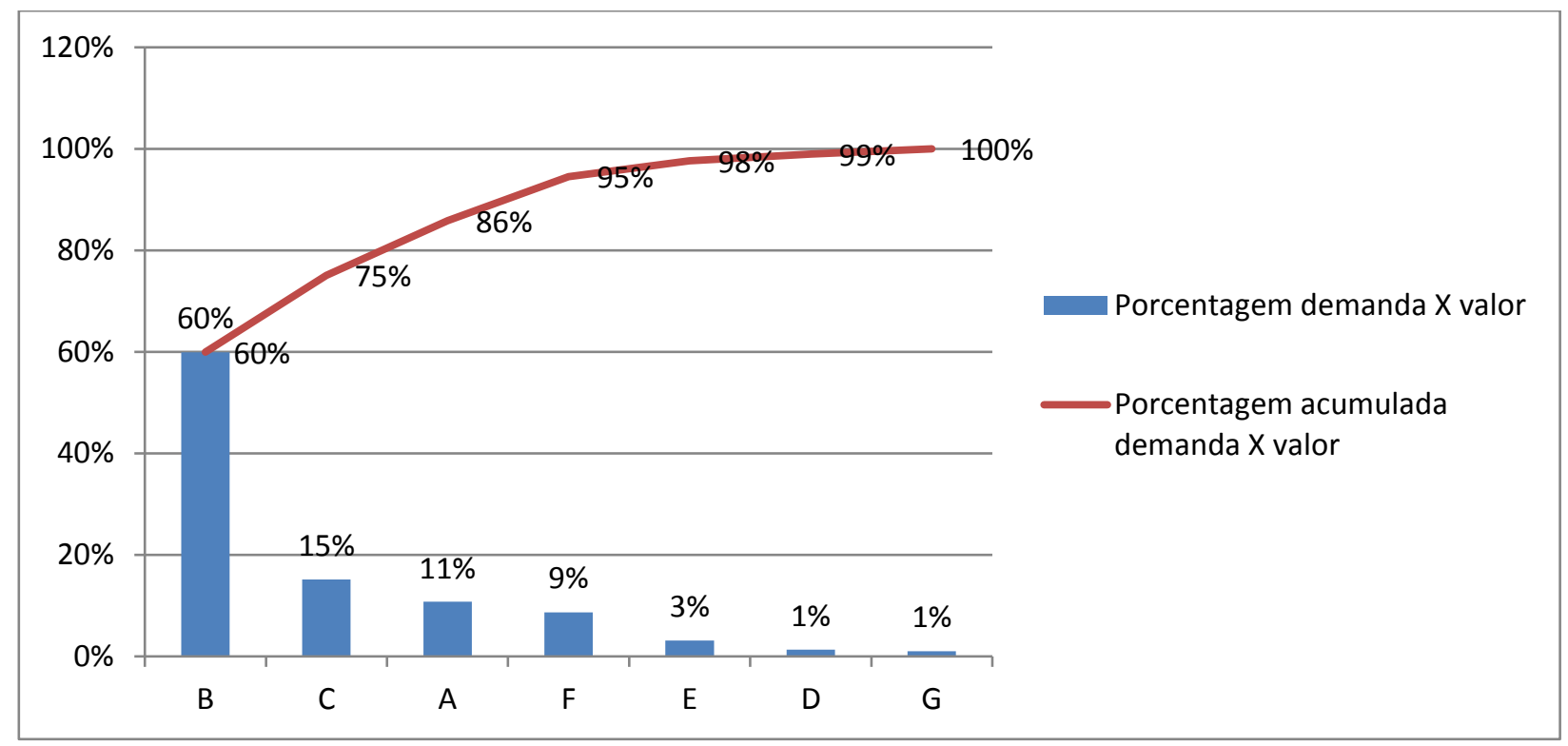

Gráfico 1. Curva da demanda multiplicada pelo valor do produto em \%.

Verifica que os itens B, C e A representam $86 \%$ do total, enquanto os itens $F, E$, D e $G$ representam os outros $14 \%$ do total. Dessa forma, focou-se o estudo nos itens $A$, $\mathrm{B}$ e $\mathrm{C}$, pois, entende-se que esses itens, pela maior rotatividade e representatividade, devem estar disponíveis no estoque para quando o cliente necessitar. $O$ mesmo não ocorre com os itens F, E, D e G, os quais são considerados uma linha especial, da qual os clientes são mais tolerantes à espera.

Eleitos os itens que a empresa deve focar para manter estoque, seguiu-se para a segunda parte da análise, onde se calculou o desvio padrão de cada um dos itens $(A, B$ e C) com a Equação 2.

De acordo com os valores de desvio padrão obtidos a partir dos dados de demanda e, calculando-se os limites inferior e superior de controle com as Equações 3 e 4, elaborou-se o Gráfico 2 que apresenta a demanda e as linhas dos limites inferior e superior de controle, considerando 3 desvios padrões em relação à média. 


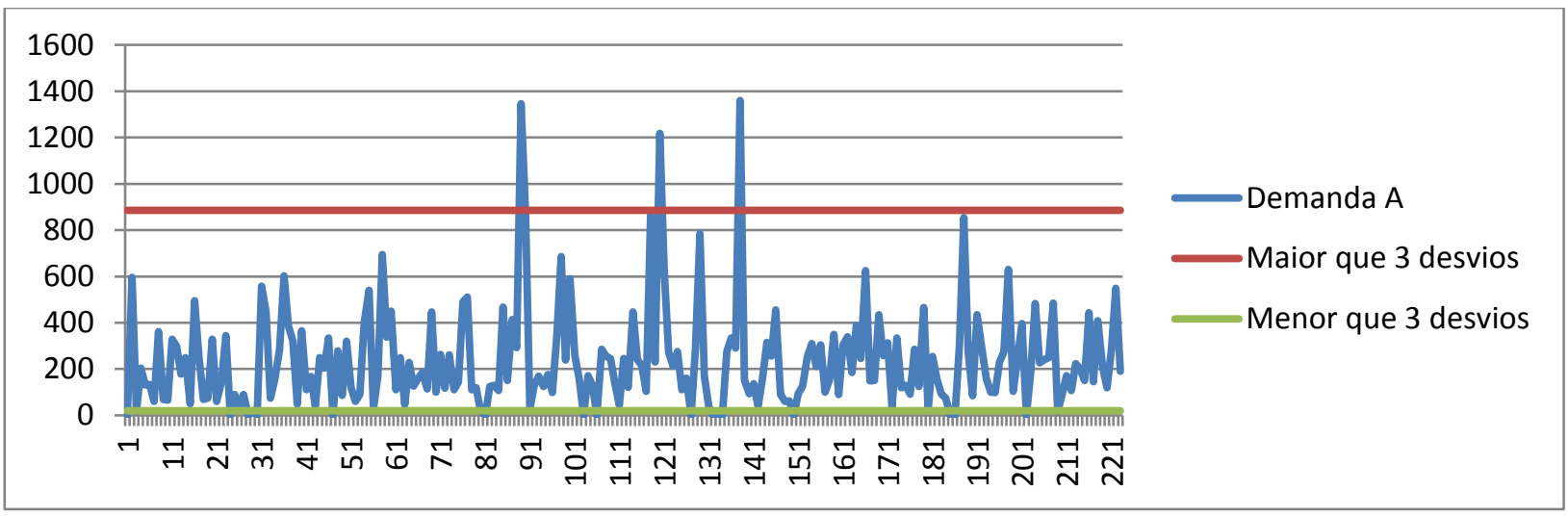

Gráfico 2. Curvas da demanda com linhas de corte dos valores maiores e menores que 3 desvios.

Os três pontos que estão acima do limite superior de controle são considerados causas especiais e não devem entrar nos cálculos (Werkema, 1995), pois, considera-se que são pedidos esporádicos e pontuais ao longo do tempo. Assim, foram excluídos para que se obtivessem os valores de demanda mais condizentes com a realidade de pedidos da empresa. O Gráfico 3 mostra a linha da demanda com esses novos valores.

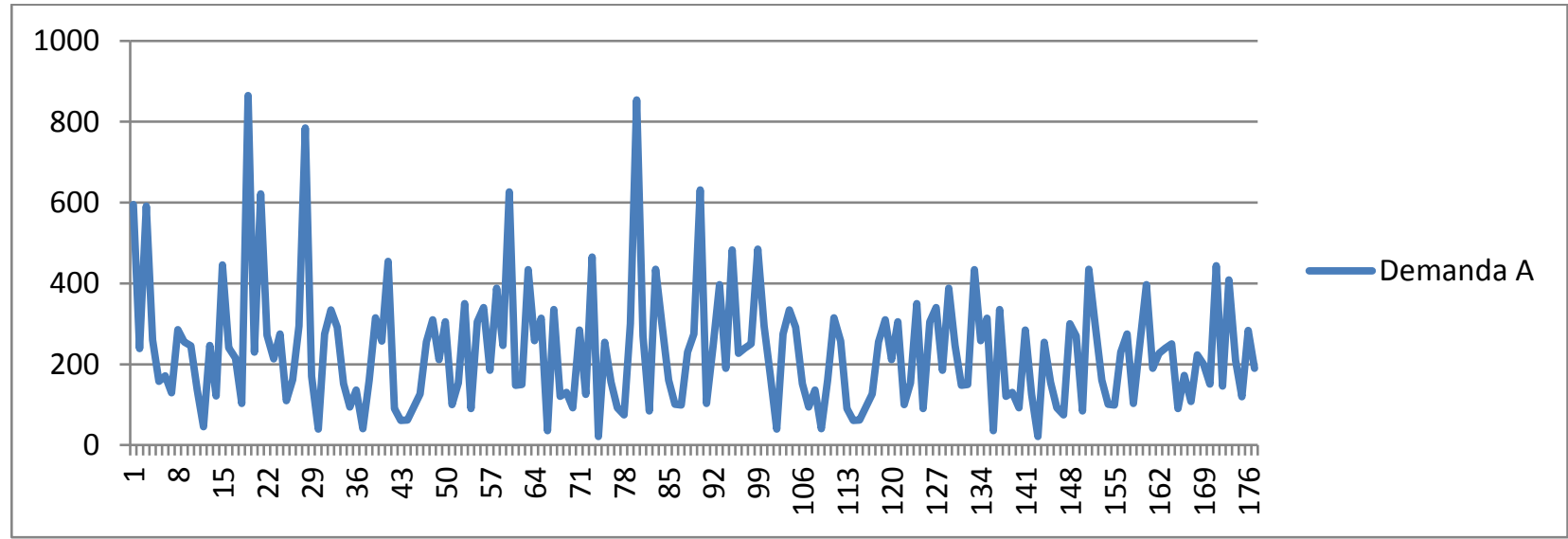

Gráfico 3. Gráfico da demanda com os valores maiores e menos que 3 desvios excluídos. 
Com esses dados, calcularam-se os valores de média (Equação 1) e seus desvios (Equação 2) novamente. Os resultados estão apresentados na Tabela 1.

Tabela 1

Dados estatísticos da demanda

\begin{tabular}{lccc}
\hline & A & B & C \\
\hline Média & 232 & 441 & 124 \\
Maior valor & 865 & 1630 & 760 \\
Menor valor & 21 & 29 & 0 \\
Desvio padrão $(\sigma)$ & 149 & 366 & 170 \\
\hline
\end{tabular}

Nota. Fonte: Dados da pesquisa.

A partir dos dados da Tabela 1, definiram-se os lotes de cada um dos itens de acordo com os desvios padrões. Os valores negativos foram zerados, pois não existe unidade de produto negativa. Somou-se e diminuiu-se o valor da média de $A, B$ e $C$ com $1 \sigma, 2 \sigma$ e $3 \sigma$ desvios. Esse procedimento foi realizado no intuito de abranger as três faixas de valores da distribuição normal. Os resultados estão descritos na Tabela 2.

Tabela 2

Valores dos lotes em relação ao desvio padrão

\begin{tabular}{cccccccc}
\hline & $\mathbf{- 3} \boldsymbol{\sigma}$ & $\mathbf{- 2} \boldsymbol{\sigma}$ & $\mathbf{- 1} \boldsymbol{\sigma}$ & $\boldsymbol{\mu} \mathbf{A}$ & $\mathbf{+ 1} \boldsymbol{\sigma}$ & $\mathbf{+ 2} \boldsymbol{\sigma}$ & $\mathbf{+ 3} \boldsymbol{\sigma}$ \\
\hline $\mathrm{A}$ & 0 & 0 & 83 & 232 & 381 & 530 & 679 \\
$\mathrm{~B}$ & 0 & 0 & 75 & 441 & 807 & 1173 & 1539 \\
$\mathrm{C}$ & 0 & 0 & 0 & 124 & 294 & 464 & 634 \\
\hline
\end{tabular}

Nota. Fonte: Dados da pesquisa.

Com os valores da Tabela 2, confrontaram-se os valores de lotes de pedidos para verificar a porcentagem de atendimento aos pedidos de clientes. A Tabela 3 apresenta esses dados. Esse procedimento foi realizado simulando os atendimentos aos clientes com os dados dos pedidos realizados no passado em relação aos novos 
lotes estabelecidos. Com esses dados, foi possível visualizar qual seria a porcentagem de clientes que a empresa arriscaria deixar de atender por não ter estoque.

Tabela 3

Valores nominais da demanda em relação à média e porcentagem de atendimento aos pedidos dos clientes

\begin{tabular}{lccc}
\hline & A & B & C \\
\hline \% Média & $53 \%$ & $57 \%$ & $65 \%$ \\
$\%$ 1 Desvio & $87 \%$ & $86 \%$ & $82 \%$ \\
$\%$ 2 Desvios & $95 \%$ & $95 \%$ & $87 \%$ \\
\% 3 Desvios & $98 \%$ & $100 \%$ & $92 \%$ \\
\hline
\end{tabular}

Nota. Fonte: Dados da pesquisa.

Com esses valores calculados, foi possível analisar o custo de atendimento ao cliente e estudar o custo de cada uma das porcentagens de atendimento ao cliente. Os valores obtidos a partir dos desvios padrão foram divididos pelos valores encontrados da média. Assim foi possível criar um fator de multiplicação entre os valores, conforme segue na Tabela 4.

Tabela 4

Relação do custo de acordo com os desvios.

\begin{tabular}{lccc}
\hline & A & B & C \\
\hline média / média & 1 & 1 & 1 \\
1 desvio / média & 1,64 & 1,83 & 2,38 \\
2 desvios / média & 2,29 & 2,66 & 3,75 \\
3 desvios / média & 2,93 & 3,49 & 5,13 \\
\hline
\end{tabular}

Nota. Fonte: Dados da pesquisa.

Os fatores apresentados na Tabela 4 relacionam o quanto o valor do custo do estoque aumenta proporcionalmente, caso a empresa opte por atender cada faixa de desvios em relação à média. 


\section{DISCUSSÕES}

Com os resultados obtidos na análise do Gráfico de Pareto, foi possível dividir os produtos em duas categorias: produtos mais representativos (A, B e C) e produtos menos representativos ( $D, E, F$ e G). Esta classificação deriva do fato de que os produtos mais representativos compreendem mais de $80 \%$ dos valores neste setor, enquanto os outros produtos, menos representativos, compreendem os outros $20 \%$ restantes.

As sugestões e comparações de valores de estoque focaram apenas os itens mais representativos, pois esses itens apresentam características de baixa sazonalidade e oscilação da demanda, podendo, portanto, utilizar as ferramentas propostas neste estudo para análise.

Já os produtos $D, E, F$ e $G$ deverão utilizar uma tratativa diferente, visto que esses produtos não apresentam demanda frequente e são menos representativos para a empresa, ficando fora do escopo de cálculos e análises.

Com os dados da relação de custo com a porcentagem de atendimento ao cliente, é possível visualizar o quanto a mais é necessário ser gasto para atender toda a demanda. Esta configuração é demonstrada nos Gráficos 4, 5 e 6 em relação aos produtos $\mathrm{A}, \mathrm{B}$ e $\mathrm{C}$ respectivamente. 


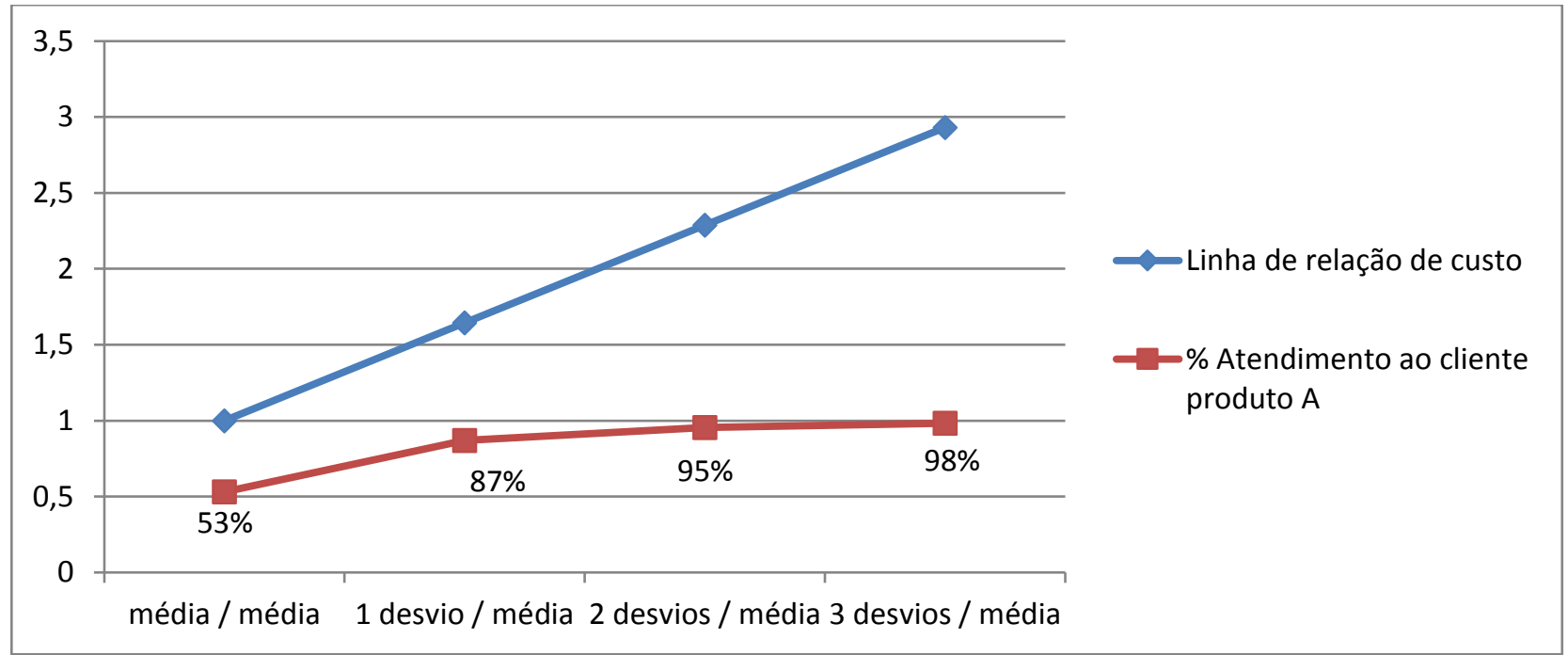

Gráfico 4. Relação de atendimento ao cliente pelo custo produto A.

O Gráfico 4 expressa a relação entre o custo e o atendimento ao cliente em relação ao produto A. Neste gráfico, é possível compreender que os valores médios encontrados nas análises de demanda atendem apenas 53\% dos clientes. Porém, 0 atendimento a $98 \%$ dos clientes onera o estoque em quase 3 vezes o seu valor.

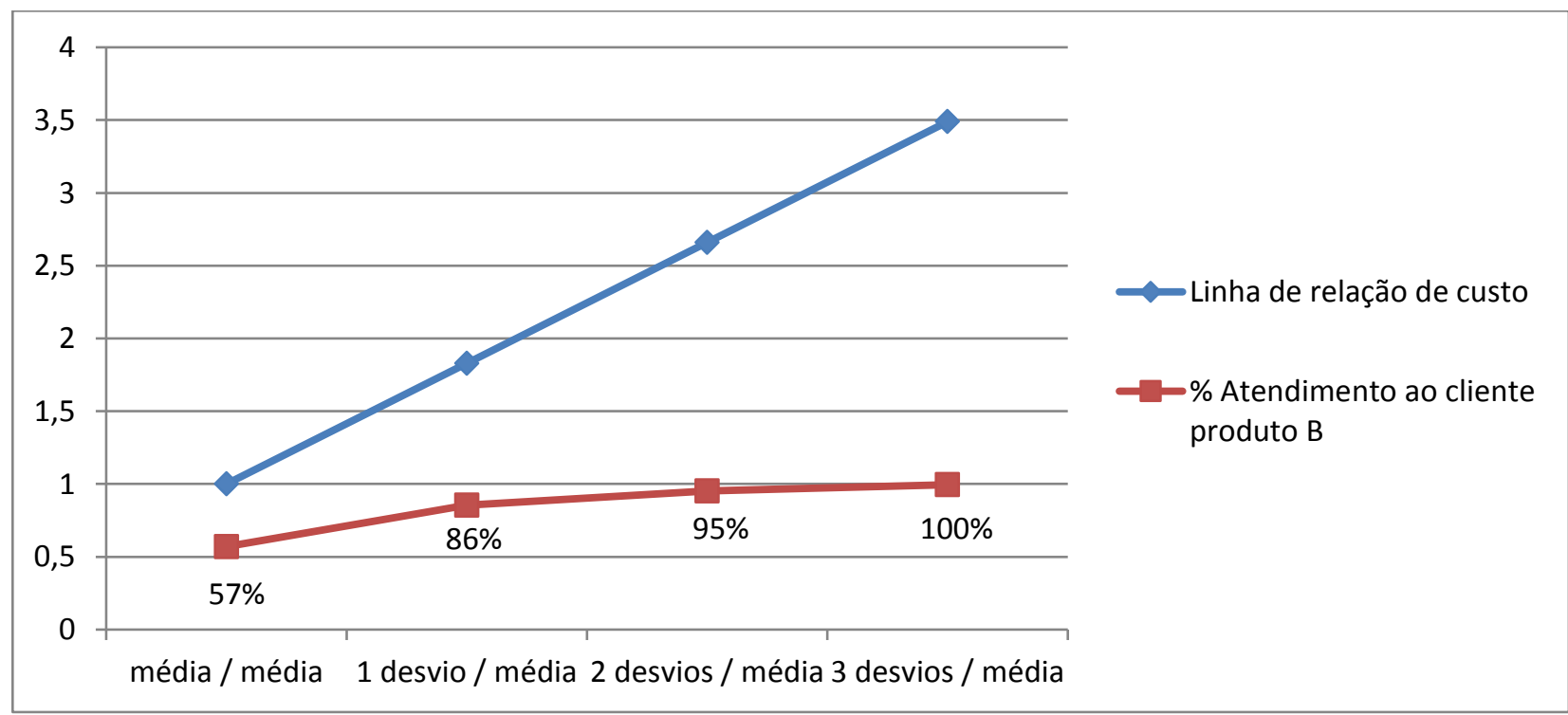

Gráfico 5. Relação de atendimento ao cliente pelo custo produto B. 
No Gráfico 5, temos a demonstração da relação entre o custo e o atendimento ao cliente em relação ao produto B. Neste caso, $57 \%$ dos clientes são atendidos quando o estoque está na média encontrada pela análise de demanda. O atendimento a $100 \%$ dos clientes aumentaria em 3,5 vezes o valor do estoque.

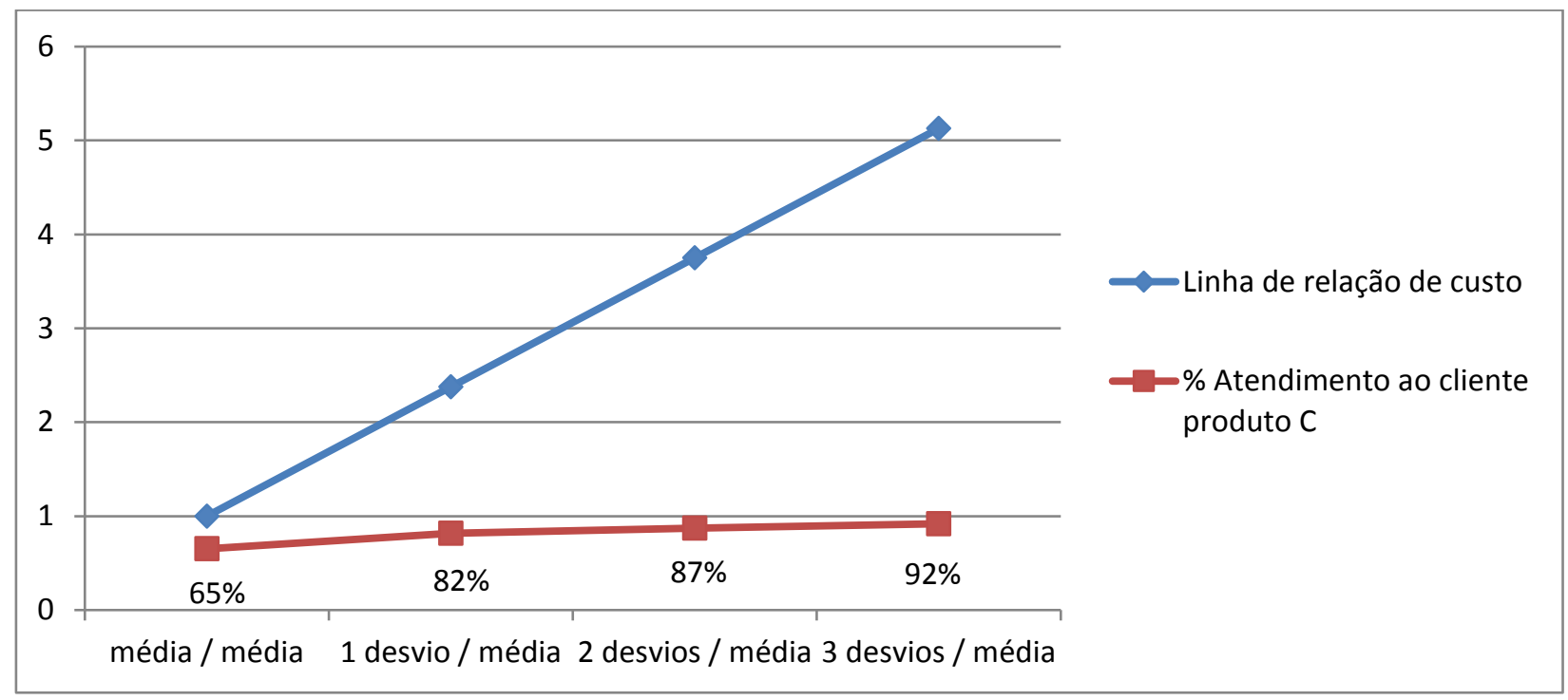

Gráfico 6. Relação de atendimento ao cliente pelo custo produto C.

No Gráfico 6, é visível o quanto o valor do estoque aumenta na medida em que tenta atender a todos os pedidos com estoque. $O$ custo chega a 5 vezes mais que 0 custo do estoque em relação ao valor médio.

Tem-se que cada empresa deve determinar sua estratégia de acordo com o mercado em que está inserida e tipo de produto que comercializa, de acordo com a criticidade do seu produto (Botter \& Fortuin, 2000). Entretanto, observa-se que o Gráfico de Pareto é uma ferramenta já bastante consolidada nos estudos científicos de estoque (Silva Júnior \& Costa, 2014; Ferrari \& Rodrigues, 2014; Carvalho \& Moura Oliveira, 2018), de forma que sua análise permite bons resultados na maioria das situações.

Porém, a análise do estoque a partir da distribuição normal traz outros entendimentos em relação ao custo e ao atendimento ao cliente, possibilitando a 
análise de outros parâmetros, como a porcentagem de atendimento ao cliente, além da representatividade do produto para a empresa.

Neste caso, a estratégia optante pela empresa foi a de atender até 2 desvios em relação à média, em que: no produto $A, 95 \%$ dos clientes são atendidos e o valor do estoque aumenta 2,3 vezes em relação à média; no produto $B, 95 \%$ dos clientes são atendidos e o valor do estoque aumenta 2,7 vezes em relação à média; e, no produto C, $87 \%$ dos clientes são atendidos e o valor do estoque aumenta em 3,7 vezes em relação à média.

Esse tipo de política de estoques implementada traz, além do benefício da disponibilidade do item em estoque para o cliente, uma maior estabilidade para a tomada de decisões dentro da própria empresa, considerando que estes valores de estoques são utilizados para a realização de pedidos de matéria-prima e para emissão de ordens de produção.

\section{CONCLUSÕES}

A partir das análises das médias e valores de desvio padrão, chegou-se na proporção em que o custo aumenta na medida em que tenta atender ao máximo o cliente. Porém, é possível equilibrar esses custos aplicando dois desvios padrões, ou seja, atendendo menos clientes, porém a maioria. E, no caso de grandes pedidos, outras políticas de negociações devem ser utilizadas.

Os principais resultados obtidos para a empresa foram uma maior clareza sobre a demanda dos seus principais produtos e seus custos, possibilitando melhores tomadas de decisões estratégicas pela empresa. Além disso, o maior controle de custos auxilia na redução de estoques segura, de modo a poder atender os clientes sem risco de não ter o produto no momento ou de ter muito estoque. Ademais, a definição de um valor ideal de estoque auxiliou a organização do trabalho do setor de programação da produção, compra de matéria-prima e vendas. 
A respeito das contribuições teóricas, tem-se que diversos estudos utilizam análises complexas que trazem uma compreensão bastante robusta sobre a realidade. Entretanto, nesta pesquisa buscou-se utilizar ferramentas simples, de fácil usabilidade, que pudessem trazer informações rápidas para possibilitar uma tomada de decisões mais breve.

Dentre as limitações, observa-se que outras análises ainda podem ser realizadas por não terem sido abarcadas no escopo deste estudo, como: analisar estratégias referentes às alianças entre fornecedores e clientes para balancear o estoque e uma tratativa diferente para a definição de estoques dos outros itens que não foram abordados nesta pesquisa por terem demanda especial.

\section{REFERÊNCIAS}

Ballou, R. H. (2004). Logística Administración de la cadena de suministro. México: Pearson Educación.

Botter, R., \& Fortuin, L. (2000). Stocking strategy for service parts - a case study, International Journal of Operations \& Production Management, 20(6), 656-674.

Carvalho, V., \& Moura Oliveira, M. (2018). Aplicação da curva de Pareto associada ao sistema Kanban para o gerenciamento de estoque numa indústria pública. Produção Em Foco, 7(2), 322-337.

Christopher, M. (2009). Logística e gerenciamento da cadeia de suprimentos. São Paulo: Cengage Learning.

Ferrari, L. C. M., \& Rodrigues, S. A. (2014). Gestão de estoque no suprimento da demanda de itens farmacêuticos de um sistema público de saúde. 3aㅡ Jornada Científica e Tecnológica da FATEC de Botucatu. São Paulo, Brasil.

Frazzon, E., Tortorella, G. L., Dávalos, R., Holtz, T., \& Coelho, T. (2017). Simulationbased analysis of a supplier-manufacturer relationship in lean supply chains, International Journal of Lean Six Sigma, 8(3), 262-274.

Huiskonen, J. (2001). Maintenance spare parts logistics: special characteristics and strategic choices. International Journal of Production Economics, 71(1), 125-133. 
Kauark, F. S., Manhães, F. C., \& Medeiros, C. H. (2010). Metodologia da pesquisa: um guia prático. Bahia: Via Literarum.

Martins, G. A., \& Domingues, O. (2011). Estatística Geral e Aplicada. São Paulo: Editora Atlas.

Moura, R. A. (2011) Armazenagem: do recebimento à expedição. São Paulo: IMAM.

Moreira, D. A. (2004) Administração da Produção e Operações. São Paulo: Pioneira Thomson Learning.

Rogers, P., Ribeiro, K. C. S., \& Rogers, D. (2004). Avaliando o risco na gestão financeira de estoque. Anais do VII Simpósio de Administração da Produção, Logística e Operações Internacionais - FGV-EAESP, Brasil.

Silva Júnior, L. J., \& Costa, F. P. (2014). Utilização da curva ABC como ferramenta de apoio ao gerenciamento de estoque em microempresa de autopeça. XXXIV Encontro Nacional de Engenharia de Produção. Curitiba, Brasil.

Womack, J, Jones, D. T., \& Roos, D. (2004). A máquina que mudou o mundo: baseado no estudo do Massachusetts Institute of Technology sobre o futuro do automóvel. (11a ed.). Rio de Janeiro: Elsevier.

Yin, R. K. (2007). Estudo de caso: planejamento e métodos. (3a ed.). Porto Alegre: Bookman.

Werkema, M. C. C. (1995). Ferramentas estatísticas básicas para o gerenciamento de processos. Belo Horizonte: Fundação Christiano Ottoni.

Data de Submissão: 28/06/2018

Data de Aceite: 17/04/2019 